\title{
The improved disc theorems for the Schur complements of diagonally dominant matrices
}

\author{
Juan Zhang ${ }^{1}$, Jianzhou Liu' ${ }^{1,2^{*}}$ and Gen Tu²
}

"Correspondence: liujz@xtu.edu.cn 'Department of Mathematics and Computational Science, Xiangtan University, Xiangtan, Hunan 411105, P.R. China

${ }^{2}$ Key Laboratory of Intelligent Computing and Information Processing of Ministry of Education, Xiangtan University, Xiangtan, Hunan 411105, P.R. China

\begin{abstract}
The theory of Schur complement is very important in many fields such as control theory and computational mathematics. In this paper, applying the properties of Schur complement, utilizing some inequality techniques, some new estimates of diagonally dominant degree on the Schur complement of matrices are obtained, which improve some relative results. Further, as an application of these derived results, we present some distributions for the eigenvalues of the Schur complements. Finally, the numerical example is given to show the advantages of our derived results. MSC: $15 \mathrm{~A} 45 ; 15 \mathrm{~A} 48$

Keywords: Schur complement; diagonally dominant matrices; eigenvalue; Gerschgorin theorem
\end{abstract}

\section{Introduction}

The Schur complement has been proved to be a useful tool in many fields such as numerical algebra and control theory. [1,2] proposed a kind of iteration called the Schur-based iteration. Applying this method, we can solve large scale linear systems though reducing the order by the Schur complement. In addition, when utilizing the conjugate gradient method to solve large scale linear systems, if the eigenvalues of the system matrix are more concentrated, the convergent speed of the iterative method is faster (see, e.g., [3, pp.312-317]). From [1, 2], it can be seen that for large scale linear systems, after applying the Schur-based iteration to reduce the order, the corresponding system matrix of linear equations is the Schur complement of the system matrix of original large scale linear systems and its eigenvalues are more concentrated than those of the original system matrix, leading to the Schur-based conjugate gradient method computing faster than the ordinary conjugate gradient method.

Hence, it is always interesting to know whether some important properties of matrices are inherited by their Schur complements. Clearly, the Schur complements of positive semidefinite matrices are positive semidefinite, the same is true for $M$-matrices, $H$-matrices and inverse $M$-matrices (see [4, 5]). Carlson and Markham showed that the Schur complements of strictly diagonally dominant matrices are diagonally dominant (see [6]). Li, Tsatsomeros and Ikramov independently proved the Schur complement of a strictly doubly diagonally dominant matrix is strictly doubly diagonally dominant (see $[7,8])$. These properties have been repeatedly used for the convergence of iterations in

(c) 2013 Zhang et al.; licensee Springer. This is an Open Access article distributed under the terms of the Creative Commons Attribution License (http://creativecommons.org/licenses/by/2.0), which permits unrestricted use, distribution, and reproduction in any medium, provided the original work is properly cited. 
numerical analysis and for deriving matrix inequalities in matrix analysis (see $[3,9,10])$. More importantly, the distribution for the eigenvalues of the Schur complement is of great significance, as shown in $[1,2,8,11-17]$. The aim of this paper is to study the distributions for the eigenvalues of the Schur complement of some diagonally dominant matrices.

Denote by $\mathbb{C}^{n \times n}$ the set of all $n \times n$ complex matrices. Let $N=\{1,2, \ldots, n\}$. For $A=\left(a_{i j}\right) \in$ $\mathbb{C}^{n \times n}(n \geq 2)$, assume

$$
P_{i}(A)=\sum_{j \in N, j \neq i}\left|a_{i j}\right|, \quad S_{i}(A)=\sum_{j \in N, j \neq i}\left|a_{j i}\right|, \quad i=1,2, \ldots, n .
$$

Set

$$
N_{r}(A)=\left\{i|i \in N,| a_{i i} \mid>P_{i}(A)\right\} ; \quad N_{c}(A)=\left\{j|j \in N,| a_{j j} \mid>S_{j}(A)\right\} .
$$

Let us recall that $A$ is a (row) diagonally dominant matrix $\left(D_{n}\right)$ if

$$
\left|a_{i i}\right| \geq P_{i}(A), \quad \forall i \in N
$$

$A$ is a doubly diagonally dominant matrix $\left(D D_{n}\right)$ if

$$
\left|a_{i i}\right|\left|a_{j j}\right| \geq P_{i}(A) P_{j}(A), \quad \forall i \neq j, i, j \in N
$$

$A$ is a $\gamma$-diagonally dominant matrix $\left(D_{n}^{\gamma}\right)$ if there exists $\gamma \in[0,1]$ such that

$$
\left|a_{i i}\right| \geq \gamma P_{i}(A)+(1-\gamma) Q_{i}(A), \quad \forall i \in N
$$

$A$ is a product $\gamma$-diagonally dominant matrix $\left(P D_{n}^{\gamma}\right)$ if there exists $\gamma \in[0,1]$ such that

$$
\left|a_{i i}\right| \geq\left[P_{i}(A)\right]^{\gamma}\left[Q_{i}(A)\right]^{1-\gamma}, \quad \forall i \in N
$$

If all inequalities in (1.1)-(1.4) are strict, then $A$ is said to be a strictly (row) diagonally dominant matrix $\left(S D_{n}\right)$, a strictly doubly diagonally dominant matrix $\left(S D D_{n}\right)$, a strictly $\gamma$-diagonally dominant matrix $\left(S D_{n}^{\gamma}\right)$ and a strictly product $\gamma$-diagonally dominant matrix $\left(S P D_{n}^{\gamma}\right)$, respectively.

Liu and Zhang in [14] have pointed out the fact as follows. If $A \in S D D_{n}$ but $A \notin S D_{n}$, then there exists a unique index $i_{0}$ such that

$$
\left|a_{i_{0} i_{0}}\right| \leq P_{i_{0}}(A) .
$$

As in $[1,2]$, for $1 \leq i \leq n$ and $\gamma \in[0,1]$, we call $\left|a_{i i}\right|-P_{i}(A),\left|a_{i i}\right|-\gamma P_{i}(A)-(1-\gamma) S_{i}(A)$ and $\left|a_{i i}\right|-\left[P_{i}(A)\right]^{\gamma}\left[S_{i}(A)\right]^{1-\gamma}$ the $i$ th (row) dominant degree, $\gamma$-dominant degree and product $\gamma$-dominant degree of $A$, respectively.

The comparison matrix of $A$, denoted by $\mu(A)=\left(t_{i j}\right)$, is defined to be

$$
t_{i j}= \begin{cases}\left|a_{i j}\right|, & \text { if } i=j, \\ -\left|a_{i j}\right|, & \text { if } i \neq j .\end{cases}
$$


A matrix $A$ is an $M$-matrix if it can be written in the form of $A=m I-P$ with $P$ being nonnegative and $m>\rho(P)$, where $\rho(P)$ denotes the spectral radius of $P$. A matrix $A$ is a $H$-matrix if $\mu(A)$ is a $M$-matrix. We denote by $\mathbb{H}_{n}$ and $\mathbb{M}_{n}$ the sets of $n \times n H$ - and $M$-matrices, respectively.

For $\alpha \subseteq N$, denote by $|\alpha|$ the cardinality of $\alpha$ and $\alpha^{\prime}=N-\alpha$. If $\alpha, \beta \subseteq N$, then $A(\alpha, \beta)$ is the submatrix of $A$ lying in the rows indicated by $\alpha$ and the columns indicated by $\beta$. In particular, $A(\alpha, \alpha)$ is abbreviated to $A(\alpha)$. Assume that $A(\alpha)$ is nonsingular. Then

$$
A / \alpha=A / A(\alpha)=A\left(\alpha^{\prime}\right)-A\left(\alpha^{\prime}, \alpha\right)[A(\alpha)]^{-1} A\left(\alpha, \alpha^{\prime}\right)
$$

is called the Schur complement of $A$ with respect to $A(\alpha)$.

The paper is organized as follows. In Section 2, we give several new estimates of diagonally dominant degree on the Schur complement of matrices, which improve some relative results. In Section 3, as an application of these derived results, the distributions for eigenvalues are obtained. In Section 4, we give a numerical example to show the advantages of our derived results.

\section{The diagonally dominant degree for the Schur complement}

In this section, we give several new estimates of diagonally dominant degree on the Schur complement of matrices, which improve some relative results.

Lemma 1 [4] If $A$ is an $H$-matrix, then $[\mu(A)]^{-1} \geq\left|A^{-1}\right|$.

Lemma 2 [4] If $A \in S D_{n}$ or $S D D_{n}$, then $\mu(A) \in \mathbb{M}_{n}$, i.e., $A \in \mathbb{H}_{n}$.

Lemma 3 [11] If $A \in S D_{n}$ or $S D D_{n}$ and $\alpha \subseteq N$, then the Schur complement of $A$ is in $S D_{\left|\alpha^{\prime}\right|}$ or $S D D_{\left|\alpha^{\prime}\right|}$, where $\alpha^{\prime}=N-\alpha$ is the Schur complement of $\alpha$ in $N$ and $\left|\alpha^{\prime}\right|$ is the cardinality of $\alpha^{\prime}$.

Lemma 4 [1] Let $a>b, c>b, b>0$ and $0 \leq r \leq 1$. Then

$$
a^{r} c^{1-r} \geq(a-b)^{r}(c-b)^{1-r}+b
$$

Theorem 1 Let $A \in \mathbb{C}^{n \times n}, \alpha=\left\{i_{1}, i_{2}, \ldots, i_{k}\right\} \subseteq N_{r}(A), \alpha^{\prime}=N-\alpha=\left\{j_{1}, j_{2}, \ldots, j_{l}\right\},|\alpha|<n$ and denote $A / \alpha=\left(a_{t s}^{\prime}\right)$. Then for all $1 \leq t \leq l$,

$$
\left|a_{t t}^{\prime}\right|-P_{t}(A / \alpha) \geq\left|a_{j_{t j} t}\right|-P_{j_{t}}(A)+w_{j_{t}} \geq\left|a_{j_{t j} t}\right|-P_{j_{t}}(A)
$$

and

$$
\left|a_{t t}^{\prime}\right|+P_{t}(A / \alpha) \leq\left|a_{j_{t} j_{t}}\right|+P_{j_{t}}(A)-w_{j_{t}} \leq\left|a_{j_{t} j_{t}}\right|+P_{j_{t}}(A)
$$

where

$$
w_{j t}=\min _{1 \leq \omega \leq k} \frac{\left|a_{i_{\omega} i_{\omega}}\right|-P_{i_{\omega}}(A)}{\left|a_{i_{\omega} i_{\omega}}\right|-\sum_{\substack{u=1 \\ u \neq \omega}}^{k}\left|a_{i_{\omega} i_{u}}\right|} \sum_{u=1}^{k}\left|a_{j t} i_{u}\right| .
$$


Proof According to Lemmas 1 and 2, we have $\{\mu[A(\alpha)]\}^{-1} \geq[A(\alpha)]^{-1}$. Thus, $\forall \varepsilon>0$ and $t=1,2, \ldots, l$,

$$
\begin{aligned}
& \left|a_{t t}^{\prime}\right|-P_{t}(A / \alpha) \\
& =\left|a_{t t}^{\prime}\right|-\sum_{\substack{s=1 \\
s \neq t}}^{l}\left|a_{t s}^{\prime}\right| \\
& =\left|a_{j t_{t}}-\left(a_{j t i_{1}}, \ldots, a_{j t_{k} i_{k}}\right)[A(\alpha)]^{-1}\left(\begin{array}{c}
a_{i_{1} j_{t}} \\
\vdots \\
a_{i_{k} j_{t}}
\end{array}\right)\right| \\
& -\sum_{\substack{s=1 \\
s \neq t}}^{l}\left|a_{j_{t} j_{s}}-\left(a_{j_{t} i_{1}}, \ldots, a_{j_{t} i_{k}}\right)[A(\alpha)]^{-1}\left(\begin{array}{c}
a_{i_{1} j_{s}} \\
\vdots \\
a_{i_{k} j_{s}}
\end{array}\right)\right| \\
& \geq\left|a_{j_{t} j_{t}}\right|-\sum_{\substack{s=1 \\
s \neq t}}^{l}\left|a_{j_{t} j_{s}}\right|-\sum_{s=1}^{l}\left|\left(a_{j_{t} i_{1}}, \ldots, a_{j_{t} i_{k}}\right)[A(\alpha)]^{-1}\left(\begin{array}{c}
a_{i_{1} j_{s}} \\
\vdots \\
a_{i_{k} j_{s}}
\end{array}\right)\right| \\
& \geq\left|a_{j t_{j} t}\right|-\sum_{\substack{s=1 \\
s \neq t}}^{l}\left|a_{j_{t} j_{s}}\right|-\sum_{s=1}^{l}\left|\left(\left|a_{j_{t} i_{1}}\right|, \ldots,\left|a_{j t i_{k}}\right|\right)\{\mu[A(\alpha)]\}^{-1}\left(\begin{array}{c}
\left|a_{i_{1} j_{s}}\right| \\
\vdots \\
\left|a_{i_{k} j_{s}}\right|
\end{array}\right)\right| \\
& \geq\left|a_{j t j_{t}}\right|-P_{j_{t}}(A)+\sum_{u=1}^{k}\left|a_{j_{t} i_{u}}\right|+\left(\omega_{j_{t}}-\varepsilon\right)-\left(\omega_{j_{t}}-\varepsilon\right) \\
& -\sum_{s=1}^{l}\left|\left(\left|a_{j t i_{1}}\right|, \ldots,\left|a_{j t i_{k}}\right|\right)\{\mu[A(\alpha)]\}^{-1}\left(\begin{array}{c}
\left|a_{i_{1} j_{s}}\right| \\
\vdots \\
\left|a_{i_{k} j_{s}}\right|
\end{array}\right)\right| \\
& \geq\left|a_{j_{t j} t}\right|-P_{j_{t}}(A)+\omega_{j_{t}}-\varepsilon+\sum_{u=1}^{k}\left|a_{j_{t} t_{u}}\right|-\omega_{j_{t}}+\varepsilon \\
& -\sum_{s=1}^{l}\left|\left(\left|a_{j_{t} i_{1}}\right|, \ldots,\left|a_{j t} i_{k}\right|\right)\{\mu[A(\alpha)]\}^{-1}\left(\begin{array}{c}
\left|a_{i_{1} j_{s}}\right| \\
\vdots \\
\left|a_{i_{k} j_{s}}\right|
\end{array}\right)\right| \\
& =\left|a_{j t j_{t}}\right|-P_{j_{t}}(A)+\omega_{j t}-\varepsilon \\
& +\frac{1}{\operatorname{det}\{\mu[A(\alpha)]\}}
\end{aligned}
$$

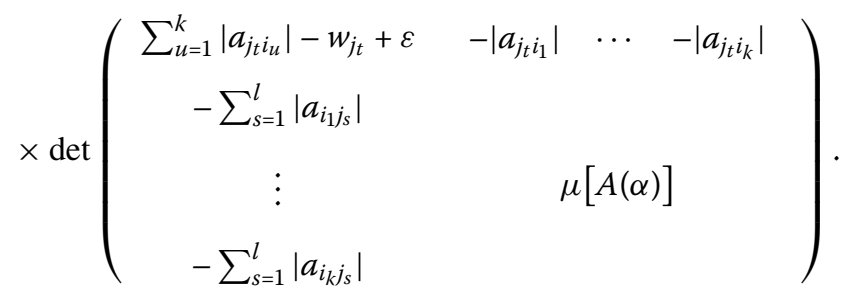


Denote

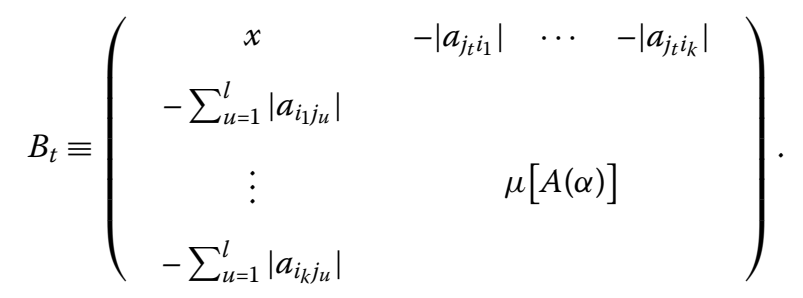

If

$$
x>\max _{1 \leq \omega \leq k} \frac{\sum_{u=1}^{l}\left|a_{i_{\omega} j_{u}}\right|}{\left|a_{i_{\omega} i_{\omega}}\right|-\sum_{\substack{u=1 \\ u \neq \omega}}^{k}\left|a_{i_{\omega} i_{u}}\right|} \sum_{u=1}^{k}\left|a_{j t i_{u}}\right|,
$$

then

$$
\frac{x}{\sum_{u=1}^{k}\left|a_{j_{t} i_{u}}\right|}>\max _{1 \leq \omega \leq k} \frac{\sum_{u=1}^{l}\left|a_{i_{\omega} j_{u}}\right|}{\left|a_{i_{\omega} i_{\omega}}\right|-\sum_{\substack{u=1 \\ u \neq \omega}}^{k}\left|a_{i_{\omega} i_{u}}\right|} .
$$

Choose $\varepsilon_{t} \in \mathbb{R}^{+}$such that

$$
\frac{x}{\sum_{u=1}^{k}\left|a_{j_{t} i_{u}}\right|}>\varepsilon_{t}>\max _{1 \leq \omega \leq k} \frac{\sum_{u=1}^{l}\left|a_{i_{\omega} j_{u}}\right|}{\left|a_{i_{\omega} i_{\omega}}\right|-\sum_{\substack{u=1 \\ u \neq \omega}}^{k}\left|a_{i_{\omega} i_{u}}\right|},
$$

where we denote $\frac{x}{\sum_{u=1}^{k}\left|a_{j_{t} t_{u}}\right|}=\infty$ if $\sum_{u=1}^{k}\left|a_{j_{t} i_{u}}\right|=0$. Set $D=\operatorname{diag}\left(y_{1}, y_{2}, \ldots, y_{k+1}\right)$, where

$$
y_{i}= \begin{cases}1, & i=1 \\ \varepsilon_{t}, & i=2,3, \ldots, k+1\end{cases}
$$

Denote $C_{t}=B_{t} D=\left(c_{s v}\right)$. If $s=1$, then

$$
\left|c_{s s}\right|-\sum_{v \neq i}\left|c_{s \nu}\right|=\left|c_{11}\right|-\sum_{s=2}^{k+1}\left|c_{1 v}\right|=x-\sum_{v=1}^{k} \varepsilon_{t}\left|a_{j t i_{v}}\right|=x-\varepsilon_{t} \sum_{v=1}^{k}\left|a_{j t} i_{v}\right|>0
$$

otherwise,

$$
\begin{aligned}
\left|c_{s s}\right|-\sum_{s \neq \nu}^{k+1}\left|c_{s v}\right| & =\varepsilon_{t}\left|a_{i_{\omega} i_{\omega}}\right|-\sum_{\nu \neq \omega}^{k} \varepsilon_{t}\left|a_{i_{\omega} i_{v}}\right|-\sum_{u=1}^{l}\left|a_{i_{\omega} j_{u}}\right| \\
& =\varepsilon_{t}\left(\left|a_{i_{\omega} i_{\omega}}\right|-\sum_{\nu \neq \omega}^{k}\left|a_{i_{\omega} i_{v}}\right|\right)-\sum_{u=1}^{l}\left|a_{i_{\omega} j_{u}}\right| \\
& >\frac{\sum_{u=1}^{l}\left|a_{i_{\omega} j_{u}}\right|}{\left|a_{i_{\omega} i_{\omega}}\right|-\sum_{\substack{u=1 \\
u \neq \omega}}^{k}\left|a_{i_{\omega} i_{u}}\right|}\left(\left|a_{i_{\omega} i_{\omega}}\right|-\sum_{\substack{u=1 \\
u \neq \omega}}^{k}\left|a_{i_{\omega} i_{u}}\right|\right)-\sum_{u=1}^{l}\left|a_{i_{\omega} j_{u}}\right|=0 .
\end{aligned}
$$


Therefore, we have $C_{t} \in S D_{k+1}$, and so $B_{t} \in \mathbb{H}_{k+1}$. Note that $B_{t}=\mu\left(B_{t}\right)$. So,

$$
\operatorname{det} B_{t}>0
$$

Take $x=\sum_{u=1}^{k}\left|a_{j_{t} i_{u}}\right|-w_{j_{t}}+\varepsilon$ in (2.5), then

$$
\begin{gathered}
\sum_{u=1}^{k}\left|a_{j_{t} i_{u}}\right|-w_{j_{t}}+\varepsilon-\max _{1 \leq \omega \leq k} \frac{\sum_{u=1}^{l}\left|a_{i_{\omega} j_{u}}\right|}{\left|a_{i_{\omega} i_{\omega}}\right|-\sum_{\substack{u=1 \\
u \neq \omega}}^{k}\left|a_{i_{\omega} i_{u}}\right|} \sum_{u=1}^{k}\left|a_{j_{t} i_{u}}\right| \\
\geq \sum_{u=1}^{k}\left|a_{j_{t} i_{u}}\right|-\min _{1 \leq \omega \leq k} \frac{\left|a_{i_{\omega} i_{\omega}}\right|-P_{i_{\omega}}(A)}{\left|a_{i_{\omega} i_{\omega}}\right|-\sum_{\substack{u=1 \\
u \neq \omega}}^{k}\left|a_{i_{\omega} i_{u}}\right|} \sum_{u=1}^{k}\left|a_{j_{t} i_{u}}\right|+\varepsilon \\
\quad-\max _{1 \leq \omega \leq k} \frac{\sum_{u=1}^{l}\left|a_{i_{\omega} j_{u}}\right|}{\left|a_{i_{\omega} i_{\omega}}\right|-\sum_{\substack{u=1 \\
u \neq \omega}}^{k}\left|a_{i_{\omega} i_{u}}\right|} \sum_{u=1}^{k}\left|a_{j t}\right|>0
\end{gathered}
$$

Noting that $\operatorname{det}\{\mu[A(\alpha)]\}>0$, by (2.4) and (2.6), we have

$$
\left|a_{t t}^{\prime}\right|-P_{t}(A / \alpha) \geq\left|a_{j_{t} j_{t}}\right|-P_{j_{t}}(A)+\omega_{j_{t}}-\varepsilon
$$

Let $\varepsilon \rightarrow 0$, thus we easily get (2.1). Similarly, we obtain (2.2).

Remark 1 Observe that

$$
\frac{P_{i_{w}}(A)}{\left|a_{i_{w} i_{w}}\right|} \geq \frac{\sum_{u=1}^{l}\left|a_{i_{\omega} j_{u}}\right|}{\left|a_{i_{\omega} i_{\omega}}\right|-\sum_{\substack{u=1 \\ u \neq \omega}}^{k}\left|a_{i_{\omega} i_{u}}\right|} .
$$

This means that Theorem 1 improves Theorem 1 of [14].

Theorem 2 Let $A \in S D D_{n}, \alpha=\left\{i_{0}, i_{1}, i_{2}, \ldots, i_{k}\right\}$ with the index $i_{0}$ satisfying (1.5), $\alpha^{\prime}=N-$ $\alpha=\left\{j_{1}, j_{2}, \ldots, j_{l}\right\},|\alpha|<n$ and denote $A / \alpha=\left(a_{t s}^{\prime}\right)$. Then for all $1 \leq t \leq l$,

$$
\begin{aligned}
\left|a_{t t}^{\prime}\right|-P_{t}(A / \alpha) & \geq\left|a_{j t j_{t}}\right|-P_{j_{t}}(A)+\left(1-\frac{\sum_{u=1}^{l}\left|a_{i_{0} j_{u}}\right|}{\left|a_{i_{0} i_{0}}\right|-\sum_{j \neq i_{0}}^{j \in \alpha}\left|a_{i_{0} j}\right|}\right) \sum_{v=1}^{k}\left|a_{j_{t} i_{v}}\right| \\
& \geq\left|a_{j_{t} j_{t}}\right|-\frac{\sum_{u=1}^{l}\left|a_{i_{0} j_{u}}\right|}{\left|a_{i_{0} i_{0}}\right|-\sum_{j \neq i_{0}}^{j \in \alpha}\left|a_{i_{0} j}\right|} P_{j_{t}}(A)
\end{aligned}
$$

and

$$
\begin{aligned}
\left|a_{t t}^{\prime}\right|+P_{t}(A / \alpha) & \leq\left|a_{j_{t} j_{t}}\right|+P_{j_{t}}(A)-\left(1-\frac{\sum_{u=1}^{l}\left|a_{i_{0} j_{u}}\right|}{\left|a_{i_{0} i_{0}}\right|-\sum_{j \neq i_{0}}^{j \in \alpha}\left|a_{i_{0} j}\right|}\right) \sum_{v=1}^{k}\left|a_{j_{t} i_{v}}\right| \\
& \leq\left|a_{j_{t} j_{t}}\right|+\frac{\sum_{u=1}^{l}\left|a_{i_{0} j_{u}}\right|}{\left|a_{i_{0} i_{0}}\right|-\sum_{j \neq i_{0}}^{j \in \alpha}\left|a_{i_{0} j}\right|} P_{j_{t}}(A) .
\end{aligned}
$$


Proof For all $1 \leq t \leq l$, we have

$$
\begin{aligned}
& \left|a_{t t}^{\prime}\right|-P_{t}(A / \alpha)=\left|a_{t t}^{\prime}\right|-\sum_{\substack{s=1 \\
s \neq t}}^{l}\left|a_{t s}^{\prime}\right| \\
& =\left|a_{j t j_{t}}-\left(a_{j t i_{1}}, \ldots, a_{j t i_{k}}\right)[A(\alpha)]^{-1}\left(\begin{array}{c}
a_{i_{1} j_{t}} \\
\vdots \\
a_{i_{k} j t}
\end{array}\right)\right| \\
& -\sum_{\substack{s=1 \\
s \neq t}}^{l}\left|a_{j_{t} j_{s}}-\left(a_{j i_{1} i_{1}}, \ldots, a_{j i_{k} i_{k}}\right)[A(\alpha)]^{-1}\left(\begin{array}{c}
a_{i_{1} j_{s}} \\
\vdots \\
a_{i_{k} j_{s}}
\end{array}\right)\right| \\
& \geq\left|a_{j t j_{t}}\right|-P_{j_{t}}(A)+\left(1-\frac{\sum_{u=1}^{l}\left|a_{i_{0} j_{u}}\right|}{\left|a_{i_{0} i_{0}}\right|-\sum_{j \neq i_{0}}^{j \in \alpha}\left|a_{i_{0} j}\right|}\right) \sum_{v=1}^{k}\left|a_{j t_{\nu}}\right| \\
& +\frac{\sum_{u=1}^{l}\left|a_{i_{0} j_{u}}\right|}{\left|a_{i_{0} i_{0}}\right|-\sum_{j \neq i_{0}}^{j \in \alpha}\left|a_{i_{0} j}\right|} \sum_{v=1}^{k}\left|a_{j t}\right| \\
& -\sum_{s=1}^{l}\left|\left(\left|a_{j t i_{1}}\right|, \ldots,\left|a_{j t k_{k}}\right|\right)\{\mu[A(\alpha)]\}^{-1}\left(\begin{array}{c}
\left|a_{i_{1} j_{s}}\right| \\
\vdots \\
\left|a_{i_{k} j_{s}}\right|
\end{array}\right)\right| .
\end{aligned}
$$

Thus,

$$
\begin{aligned}
& \left|a_{t t}^{\prime}\right|-P_{t}(A / \alpha) \\
& \geq\left|a_{j t j_{t}}\right|-P_{j_{t}}(A)+\left(1-\frac{\sum_{u=1}^{l}\left|a_{i_{0} j_{u}}\right|}{\left|a_{i_{0} i_{0}}\right|-\sum_{\substack{j \in i_{0} \\
j \in \alpha}}\left|a_{i_{0} j}\right|}\right) \sum_{v=1}^{k}\left|a_{j t i_{v}}\right|
\end{aligned}
$$

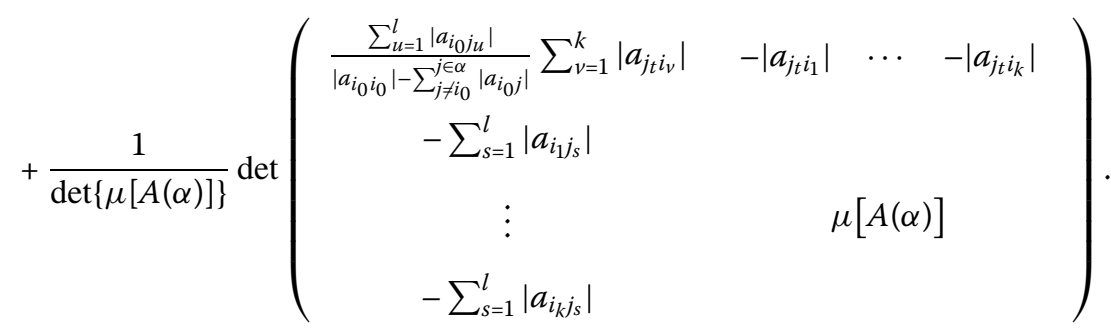

Take $x=\frac{\sum_{u=1}^{l}\left|a_{i_{0} j_{u}}\right|}{\left|a_{i_{0} i_{0}}\right|-\sum_{j \neq i_{0}}^{j \in \alpha}\left|a_{i_{0}}\right|} \sum_{v=1}^{k}\left|a_{j t i_{v}}\right|$ in (2.5). By (2.6), thus it is not difficult to get that (2.7) follows. Similarly, we obtain (2.8).

It is known that the Schur complements of diagonally dominant matrices are diagonally dominant (see $[12,13])$. However, this property is not always true for $\gamma$-diagonally dominant matrices and for product $\gamma$-diagonally dominant matrices, as shown in [1].

In the sequel, we obtain some disc separations for the $\gamma$-diagonally and product $\gamma$ diagonally dominant degree of the Schur complement, from which we provide that the Schur complement of the $\gamma$-diagonally and product $\gamma$-diagonally dominant matrices is also $\gamma$-diagonally dominant and product $\gamma$-diagonally under some restrictive conditions. 
Theorem 3 Let $A \in \mathbb{C}^{n \times n}, \alpha=\left\{i_{1}, i_{2}, \ldots, i_{k}\right\} \subseteq N_{r}(A) \cap N_{c}(A) \neq \emptyset, \alpha^{\prime}=N-\alpha=\left\{j_{1}, j_{2}, \ldots, j_{l}\right\}$, $|\alpha|<n$ and denote $A / \alpha=\left(a_{t s}^{\prime}\right)$. Then for all $1 \leq t \leq l$,

$$
\begin{aligned}
\left|a_{t t}^{\prime}\right|-P_{t}^{\gamma}(A / \alpha) S_{t}^{(1-\gamma)}(A / \alpha) & >\left|a_{j_{t j} j_{t}}\right|-\left(P_{j_{t}}(A)-w_{t}\right)^{\gamma}\left(S_{j_{t}}(A)-w_{t}^{T}\right)^{1-\gamma} \\
& >\left|a_{j t j_{t}}\right|-P_{j_{t}}^{\gamma}(A) S_{j_{t}}^{1-\gamma}(A)
\end{aligned}
$$

and

$$
\begin{aligned}
\left|a_{t t}^{\prime}\right|+P_{t}^{\gamma}(A / \alpha) S_{t}^{(1-\gamma)}(A / \alpha) & <\left|a_{j t j_{t}}\right|+\left(P_{j_{t}}(A)-w_{t}\right)^{\gamma}\left(S_{j_{t}}(A)-w_{t}^{T}\right)^{1-\gamma} \\
& <\left|a_{j t j_{t}}\right|+P_{j_{t}}^{\gamma}(A) S_{j_{t}}^{1-\gamma}(A)
\end{aligned}
$$

where

$$
\begin{aligned}
& w_{t}=\min _{1 \leq v \leq k} \frac{\left|a_{i_{v} i_{v}}\right|-P_{i_{v}}(A)}{\left|a_{i_{\nu} i_{v}}\right|-\sum_{\substack{u=1 \\
u \neq v}}^{k}\left|a_{i_{v} i_{u}}\right|} \sum_{u=1}^{k}\left|a_{j t} i_{u}\right| ; \\
& w_{t}^{T}=\min _{1 \leq \nu \leq k} \frac{\left|a_{i_{\nu} i_{v}}\right|-S_{i_{v}}(A)}{\left|a_{i_{v} i_{v}}\right|-\sum_{\substack{u=1 \\
u \neq v}}^{k}\left|a_{i_{u} i_{v}}\right|} \sum_{u=1}^{k}\left|a_{i_{u} j}\right| .
\end{aligned}
$$

Proof For all $1 \leq t \leq l$, we have

$$
\begin{aligned}
& \left|a_{t t}^{\prime}\right|-P_{t}^{\gamma}(A / \alpha) S_{t}^{(1-\gamma)}(A / \alpha) \\
& =\left|a_{t t}^{\prime}\right|-\left(\sum_{\substack{s=1 \\
s \neq t}}^{l}\left|a_{t s}^{\prime}\right|\right)^{\gamma}\left(\sum_{\substack{s=1 \\
s \neq t}}^{l}\left|a_{s t}^{\prime}\right|\right)^{1-\gamma} \\
& =\left|a_{j t j_{t}}-\left(a_{j t i_{1}}, \ldots, a_{j t i_{k}}\right)[A(\alpha)]^{-1}\left(\begin{array}{c}
a_{i_{1} j_{t}} \\
\vdots \\
a_{i_{k} j t}
\end{array}\right)\right| \\
& -\left[\sum_{\substack{s=1 \\
s \neq t}}^{l}\left|a_{j t j_{s}}-\left(a_{j t_{1}}, \ldots, a_{j_{t} i_{k}}\right)[A(\alpha)]^{-1}\left(\begin{array}{c}
a_{i_{1} j_{s}} \\
\vdots \\
a_{i_{k} j_{s}}
\end{array}\right)\right|\right]^{\gamma} \\
& \times\left[\sum_{\substack{s=1 \\
s \neq t}}^{l}\left|a_{j_{s} j_{t}}-\left(a_{j_{s} i_{1}}, \ldots, a_{j_{s} i_{k}}\right)[A(\alpha)]^{-1}\left(\begin{array}{c}
a_{i_{1} j_{t}} \\
\vdots \\
a_{i_{k} j_{t}}
\end{array}\right)\right|\right]^{(1-\gamma)} \\
& \geq\left|a_{j_{t} j_{t}}\right|-\left|\left(a_{j_{t} i_{1}}, \ldots, a_{j_{t} i_{k}}\right)[A(\alpha)]^{-1}\left(\begin{array}{c}
a_{i_{1} j_{t}} \\
\vdots \\
a_{i_{k j} j_{t}}
\end{array}\right)\right| \\
& \left.-\left(\sum_{\substack{s=1 \\
s \neq t}}^{l}\left[\left|a_{j t j_{s}}\right|+\mid\left(a_{j t i_{1}}, \ldots, a_{j t i_{k}}\right)[A(\alpha)]^{-1}\left(\begin{array}{c}
a_{i_{1} j_{s}} \\
\vdots \\
a_{i_{k} j_{s}}
\end{array}\right)\right]\right]\right)^{\gamma}
\end{aligned}
$$




$$
\begin{aligned}
& \times\left(\sum_{\substack{s=1 \\
s \neq t}}^{l}\left[\left|a_{j s j t}\right|+\left|\left(a_{j_{s} i_{1}}, \ldots, a_{j_{s} i_{k}}\right)[A(\alpha)]^{-1}\left(\begin{array}{c}
a_{i_{1} j_{t}} \\
\vdots \\
a_{i_{k} j_{t}}
\end{array}\right)\right|\right]\right)^{1-\gamma} \\
& \geq\left|a_{j t j_{t}}\right|-\left(\left|a_{j t i_{1}}\right|, \ldots,\left|a_{j t i_{k}}\right|\right)\{\mu[A(\alpha)]\}^{-1}\left(\begin{array}{c}
\left|a_{i_{1} j_{t}}\right| \\
\vdots \\
\left|a_{i_{k} j_{t}}\right|
\end{array}\right) \\
& -\left(\sum_{\substack{s=1 \\
s \neq t}}^{l}\left[\left|a_{j t_{j} s}\right|+\left(\left|a_{j t_{1}}\right|, \ldots,\left|a_{j t}\right| i_{k} \mid\right)\{\mu[A(\alpha)]\}^{-1}\left(\begin{array}{c}
\left|a_{i_{1} j_{s}}\right| \\
\vdots \\
\left|a_{i_{k} j_{s}}\right|
\end{array}\right)\right]\right)^{\gamma} \\
& \times\left(\sum_{\substack{s=1 \\
s \neq t}}^{l}\left[\left|a_{j_{s} j_{t}}\right|+\left(\left|a_{j_{s} i_{1}}\right|, \ldots,\left|a_{j_{s} i_{k}}\right|\right)\{\mu[A(\alpha)]\}^{-1}\left(\begin{array}{c}
\left|a_{i_{1} j_{t}}\right| \\
\vdots \\
\left|a_{i_{k} j_{t}}\right|
\end{array}\right)\right]\right)^{1-\gamma} .
\end{aligned}
$$

Similar as in the proof of Theorem 1, we easily obtain

$$
\begin{aligned}
& \sum_{\substack{s=1 \\
s \neq t}}^{l}\left[\left|a_{j t j_{s}}\right|+\left(\left|a_{j t i_{1}}\right|, \ldots,\left|a_{j t_{k} i_{k}}\right|\right)\{\mu[A(\alpha)]\}^{-1}\left(\begin{array}{c}
\left|a_{i_{1} j_{s}}\right| \\
\vdots \\
\left|a_{i_{k} j_{s}}\right|
\end{array}\right)\right] \\
& \quad<P_{j_{t}}(A)-w_{t}-\left(\left|a_{j t i_{1}}\right|, \ldots,\left|a_{j t i_{k}}\right|\right)\{\mu[A(\alpha)]\}^{-1}\left(\begin{array}{c}
\left|a_{i_{1} j_{t}}\right| \\
\vdots \\
\left|a_{i_{k} j_{t}}\right|
\end{array}\right) .
\end{aligned}
$$

Similarly,

$$
\begin{aligned}
& \sum_{\substack{s=1 \\
s \neq t}}^{l}\left[\left|a_{j_{s} t}\right|+\left(\left|a_{j_{s} i_{1}}\right|, \ldots,\left|a_{j_{s} i_{k}}\right|\right)\{\mu[A(\alpha)]\}^{-1}\left(\begin{array}{c}
\left|a_{i_{1} j_{t}}\right| \\
\vdots \\
\left|a_{i_{k} j_{t}}\right|
\end{array}\right)\right] \\
& \quad<S_{j_{t}}(A)-w_{t}^{T}-\left(\left|a_{j_{t} i_{1}}\right|, \ldots,\left|a_{j_{t} i_{k}}\right|\right)\{\mu[A(\alpha)]\}^{-1}\left(\begin{array}{c}
\left|a_{i_{1} j_{t}}\right| \\
\vdots \\
\left|a_{i_{k} j_{t}}\right|
\end{array}\right) .
\end{aligned}
$$

Set

$$
h=\left(\left|a_{j t_{1} i_{1}}\right|, \ldots,\left|a_{j t_{k} i_{k}}\right|\right)\{\mu[A(\alpha)]\}^{-1}\left(\begin{array}{c}
\left|a_{i_{1} j_{t}}\right| \\
\vdots \\
\left|a_{i_{k} j_{t}}\right|
\end{array}\right) .
$$

From (2.11), (2.12), (2.13), using Lemma 4, we have

$$
\begin{aligned}
& \left|a_{t t}^{\prime}\right|-P_{t}^{\gamma}(A / \alpha) S_{t}^{(1-\gamma)}(A / \alpha) \\
& \quad>\left|a_{j_{t j} j_{t}}\right|-h-\left(P_{j_{t}}(A)-w_{t}-h\right)^{\gamma}\left(S_{j_{t}}(A)-w_{t}^{T}-h\right)^{1-\gamma}
\end{aligned}
$$




$$
\begin{aligned}
& >\left|a_{j j_{j} t}\right|-h-\left[\left(P_{j_{t}}(A)-w_{t}\right)^{\gamma}\left(S_{j t}(A)-w_{t}^{T}\right)^{1-\gamma}-h\right] \\
& =\left|a_{j t j_{t}}\right|-\left(P_{j t}(A)-w_{t}\right)^{\gamma}\left(S_{j_{t}}(A)-w_{t}^{T}\right)^{1-\gamma} .
\end{aligned}
$$

Thus we get (2.9). Similarly, we have (2.10).

Remark 2 Observe that

$$
\begin{aligned}
& w_{j_{t}}=\min _{1 \leq \nu \leq k} \frac{\left|a_{i_{v} i_{v}}\right|-P_{i_{v}}(A)}{\left|a_{i_{v} i_{v}}\right|-\sum_{\substack{u=1 \\
u \neq v}}^{k}\left|a_{i_{v} i_{u}}\right|} \sum_{u=1}^{k}\left|a_{j t i_{u}}\right| \geq \min _{1 \leq v \leq k} \frac{\left|a_{i_{\nu} i_{v}}\right|-P_{i_{v}}(A)}{\left|a_{i_{v} i_{v}}\right|} \sum_{u=1}^{k}\left|a_{j t i_{u}}\right|,
\end{aligned}
$$

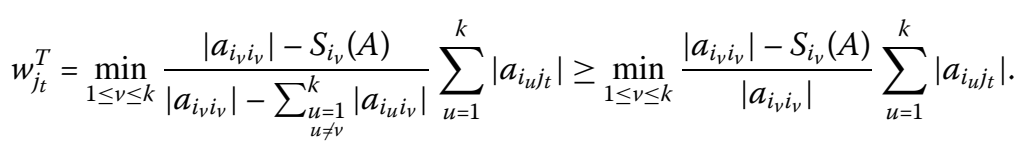

This means that Theorem 3 improves Theorem 2 of [1].

In a similar way to the proof of Theorem 3, we get the following theorem immediately.

Theorem 4 Let $A \in \mathbb{C}^{n \times n}, \alpha=\left\{i_{1}, i_{2}, \ldots, i_{k}\right\} \subseteq N_{r}(A) \cap N_{c}(A) \neq \emptyset, \alpha^{\prime}=N-\alpha=\left\{j_{1}, j_{2}, \ldots, j_{l}\right\}$, $|\alpha|<n$ and denote $A / \alpha=\left(a_{t s}^{\prime}\right)$. Then for all $1 \leq t \leq l$,

$$
\begin{aligned}
& \left|a_{t t}^{\prime}\right|-\gamma P_{t}(A / \alpha)-(1-\gamma) S_{t}(A / \alpha) \\
& \quad>\left|a_{j t j_{t}}\right|-\gamma P_{j_{t}}(A)-(1-\gamma) S_{j t}(A)+\gamma w_{t}+(1-\gamma) w_{t}^{T} \\
& \quad>\left|a_{j t j_{t}}\right|-\gamma P_{j_{t}}(A)-(1-\gamma) S_{j t}(A)
\end{aligned}
$$

and

$$
\begin{aligned}
& \left|a_{t t}^{\prime}\right|+\gamma P_{t}(A / \alpha)+(1-\gamma) S_{t}(A / \alpha) \\
& \quad<\left|a_{j j_{t} j_{t}}\right|+\gamma P_{j_{t}}(A)+(1-\gamma) S_{j_{t}}(A)-\gamma w_{t}-(1-\gamma) w_{t}^{T} \\
& \quad<\left|a_{j j_{t} j_{t}}\right|+\gamma P_{j_{t}}(A)+(1-\gamma) S_{j_{t}}(A) .
\end{aligned}
$$

Corollary 1 Let $A \in D^{\gamma}$ and $N_{r}(A) \cap N_{c}(A) \neq \emptyset$. Then for any $\alpha \subseteq N_{r}(A) \cap N_{c}(A)$ with $|\alpha|<n$,

$$
A / \alpha \in S D_{n-|\alpha|}^{\gamma}
$$

Proof By (2.14), we have

$$
\left|a_{t t}^{\prime}\right|-\gamma P_{t}(A / \alpha)-(1-\gamma) S_{t}(A / \alpha)>\left|a_{j t j_{t}}\right|-\gamma P_{j_{t}}(A)-(1-\gamma) S_{j_{t}}(A) \geq 0 .
$$

Corollary 2 Let $A \in P D_{n}^{\gamma}$ and $N_{r}(A) \cap N_{c}(A) \neq \emptyset$. Then for any $\alpha \subseteq N_{r}(A) \cap N_{c}(A)$ with $|\alpha|<n$,

$$
A / \alpha \in S P D_{n-|\alpha|}^{\gamma} .
$$




\section{Distribution for eigenvalues}

In this section, as an application of our results in Section 2, we present some locations for the eigenvalues of the Schur complements.

Theorem 5 Let $A \in \mathbb{C}^{n \times n}, \alpha=\left\{i_{1}, i_{2}, \ldots, i_{k}\right\} \subset N_{r} \neq \emptyset, \alpha^{\prime}=N-\alpha=\left\{j_{1}, j_{2}, \ldots, j_{l}\right\}$. Then for each eigenvalue $\lambda$ of $A / \alpha$, there exists $1 \leq t \leq l$ such that

$$
\left|\lambda-a_{j_{t} j_{t}}\right|<P_{j_{t}}(A)-w_{j_{t}} .
$$

Proof Set $A / \alpha=\left(a_{s t}^{\prime}\right)$. Using the Gerschgorin circle theorem, we know there exists $1 \leq t \leq l$ such that

$$
\left|\lambda-a_{t t}^{\prime}\right| \leq P_{t}(A / \alpha)
$$

Thus

$$
\begin{aligned}
& 0 \geq\left|\lambda-a_{t t}^{\prime}\right|-P_{t}(A / \alpha) \\
& =\left|\lambda-a_{j t j_{t}}+\left(a_{j t i_{1}}, \ldots, a_{j t i_{k}}\right)[A(\alpha)]^{-1}\left(\begin{array}{c}
a_{i_{1} j_{t}} \\
\vdots \\
a_{i_{k} j t}
\end{array}\right)\right| \\
& -\sum_{\substack{s=1 \\
s \neq t}}^{l}\left|a_{j t j_{s}}-\left(a_{j_{t} i_{1}}, \ldots, a_{j_{t} i_{k}}\right)[A(\alpha)]^{-1}\left(\begin{array}{c}
a_{i_{1} j_{s}} \\
\vdots \\
a_{i_{k} j_{s}}
\end{array}\right)\right| \\
& \geq\left|\lambda-a_{j t_{t} t}\right|-\sum_{\substack{s=1 \\
s \neq t}}^{l}\left|a_{j_{t} t_{s}}\right|-\sum_{s=1}^{l}\left|\left(a_{j i_{1}}, \ldots, a_{j t i_{k}}\right)[A(\alpha)]^{-1}\left(\begin{array}{c}
a_{i_{1} j_{s}} \\
\vdots \\
a_{i_{k} j_{s}}
\end{array}\right)\right| \\
& \geq\left|\lambda-a_{j_{t} t}\right|-\sum_{\substack{s=1 \\
s \neq t}}^{l}\left|a_{j_{t} j_{s}}\right|-\sum_{s=1}^{l}\left(\left|a_{j_{t} i_{1}}\right|, \ldots,\left|a_{j_{t} i_{k}}\right|\right)\{\mu[A(\alpha)]\}^{-1}\left(\begin{array}{c}
\left|a_{i_{1} j_{s}}\right| \\
\vdots \\
\left|a_{i_{k} j_{s}}\right|
\end{array}\right)
\end{aligned}
$$

(by Lemmas 1 and 2)

$$
\begin{aligned}
= & \left|\lambda-a_{j_{t} j_{t}}\right|-P_{j_{t}}(A)+\sum_{u=1}^{k}\left|a_{j_{t} i_{u}}\right|+w_{j_{t}}-w_{j_{t}} \\
& -\sum_{s=1}^{l}\left(\left|a_{j_{t} i_{1}}\right|, \ldots,\left|a_{j_{t} i_{k}}\right|\right)\{\mu[A(\alpha)]\}^{-1}\left(\begin{array}{c}
\left|a_{i_{1} j_{s}}\right| \\
\vdots \\
\left|a_{i_{k} j_{s}}\right|
\end{array}\right) \\
= & \left|\lambda-a_{j_{t j} j_{t}}\right|-P_{j_{t}}(A)+w_{j_{t}} \\
& +\sum_{u=1}^{k}\left|a_{j_{t} i_{u}}\right|-w_{j_{t}}-\left(\left|a_{j_{t} i_{1}}\right|, \ldots,\left|a_{j t_{i} i_{k}}\right|\right)\{\mu[A(\alpha)]\}^{-1}\left(\begin{array}{c}
\sum_{s=1}^{l}\left|a_{i_{1} j_{s}}\right| \\
\vdots \\
\sum_{s=1}^{l}\left|a_{i_{k} j_{s}}\right|
\end{array}\right) \\
= & \left|\lambda-a_{j_{t} j_{t}}\right|-P_{j_{t}}(A)+w_{j_{t}}
\end{aligned}
$$




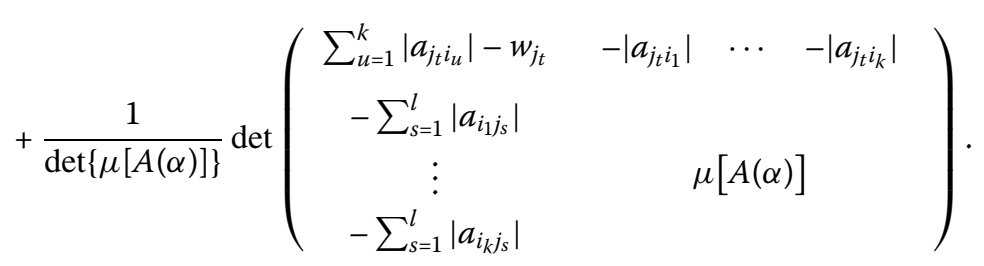

Hence, it is not difficult to get by (2.6) that

$$
0 \geq\left|\lambda-a_{t t}^{\prime}\right|-P_{t}(A / \alpha)>\left|\lambda-a_{j t j_{t}}\right|-P_{j t}(A)+w_{j t},
$$

i.e.,

$$
\left|\lambda-a_{j t j_{t}}\right|<P_{j_{t}}(A)-w_{j_{t}}
$$

Remark 3 By Remark 2, it is obvious that Theorem 5 improves Theorem 3 of [1].

Corollary 3 Let $A \in \mathbb{C}^{n \times n}, \alpha=\left\{i_{1}, i_{2}, \ldots, i_{k}\right\} \subset N_{r} \neq \emptyset, \alpha^{\prime}=N-\alpha=\left\{j_{1}, j_{2}, \ldots, j_{l}\right\}$. Then for each eigenvalue $\lambda$ of $A / \alpha$, there exists $1 \leq t \leq l$ such that

$$
\left|\lambda-a_{j_{t} j_{t}}\right|<P_{j_{t}}(A)
$$

In a similar way to the proof of Theorem 5, we obtain the following theorem according to Theorem 2.

Theorem 6 Let $A \in S D D_{n}, \alpha=\left\{i_{0}, i_{1}, i_{2}, \ldots, i_{k}\right\}$ with the index $i_{0}$ satisfying (1.5), $\alpha^{\prime}=N-$ $\alpha=\left\{j_{1}, j_{2}, \ldots, j_{l}\right\}$. Then for each eigenvalue $\lambda$ of $A / \alpha$, there exists $1 \leq t \leq l$ such that

$$
\left|\lambda-a_{j t_{t} j_{t}}\right|<P_{j_{t}}(A)-\left(1-\frac{\sum_{u=1}^{l}\left|a_{i_{0} j_{u}}\right|}{\left|a_{i_{0} i_{0}}\right|-\sum_{\substack{j \neq i_{0} \\ j \in \alpha}}\left|a_{i_{0} j}\right|}\right) \sum_{\nu=1}^{k}\left|a_{j t_{\nu}}\right| .
$$

Next, we obtain some distributions for the eigenvalues of the Schur complements of matrices under the conditions such as $P D_{n}^{\gamma}$ degree.

Lemma 5 [1] Let $A \in \mathbb{C}^{n \times n}$ and $0 \leq \gamma \leq 1$. Then for every eigenvalue $\lambda$ of $A$, there exists $1 \leq i \leq n$ such that

$$
\left|\lambda-a_{i i}\right| \leq P_{i}^{\gamma}(A) S_{i}^{1-\gamma}(A)
$$

Theorem 7 Let $A \in \mathbb{C}^{n \times n}, \alpha=\left\{i_{1}, i_{2}, \ldots, i_{k}\right\} \subseteq N_{r}(A) \cap N_{c}(A) \neq \emptyset, \alpha^{\prime}=N-\alpha=\left\{j_{1}, j_{2}, \ldots, j_{l}\right\}$, $|\alpha|<n$. Then for each eigenvalue $\lambda$ of $A / \alpha$, there exists $1 \leq t \leq l$ such that

$$
\left|\lambda-a_{j_{t} j_{t}}\right|<\left(P_{j_{t}}(A)-w_{t}\right)^{\gamma}\left(S_{j_{t}}(A)-w_{t}^{T}\right)^{1-\gamma} .
$$

Proof Set $A / \alpha=\left(a_{t s}^{\prime}\right)$. From Lemma 5, we know that for each eigenvalue $\lambda$ of $A / \alpha$, there exists $1 \leq t \leq l$ such that

$$
\left|\lambda-a_{t t}^{\prime}\right| \leq P_{t}^{\gamma}(A / \alpha) S_{t}^{1-\gamma}(A / \alpha)
$$


Hence,

$$
\begin{aligned}
& 0 \geq\left|\lambda-a_{t t}^{\prime}\right|-P_{t}^{\gamma}(A / \alpha) S_{t}^{(1-\gamma)}(A / \alpha) \\
& =\left|\lambda-a_{t t}^{\prime}\right|-\left(\sum_{\substack{s=1 \\
s \neq t}}^{l}\left|a_{t s}^{\prime}\right|\right)^{\gamma}\left(\sum_{\substack{s=1 \\
s \neq t}}^{l}\left|a_{s t}^{\prime}\right|\right)^{1-\gamma} \\
& =\left|\lambda-a_{j_{t} j_{t}}+\left(a_{j_{t} i_{1}}, \ldots, a_{j_{t} i_{k}}\right)[A(\alpha)]^{-1}\left(\begin{array}{c}
a_{i_{1} j_{t}} \\
\vdots \\
a_{i_{k} j_{t}}
\end{array}\right)\right| \\
& -\left[\sum_{\substack{s=1 \\
s \neq t}}^{l}\left|a_{j_{t} j_{s}}-\left(a_{j_{t} i_{1}}, \ldots, a_{j_{t} i_{k}}\right)[A(\alpha)]^{-1}\left(\begin{array}{c}
a_{i_{1} j_{s}} \\
\vdots \\
a_{i_{k} j_{s}}
\end{array}\right)\right|\right]^{\gamma} \\
& \times\left[\sum_{\substack{s=1 \\
s \neq t}}^{l}\left|a_{j_{s} j_{t}}-\left(a_{j_{s} i_{1}}, \ldots, a_{j_{s} i_{k}}\right)[A(\alpha)]^{-1}\left(\begin{array}{c}
a_{i_{1} j_{t}} \\
\vdots \\
a_{i_{k} j_{t}}
\end{array}\right)\right|\right]^{(1-\gamma)} \\
& \geq\left|\lambda-a_{j_{t j t}}\right|-\left|\left(a_{j_{t} i_{1}}, \ldots, a_{j_{t} i_{k}}\right)[A(\alpha)]^{-1}\left(\begin{array}{c}
a_{i_{1} j_{t}} \\
\vdots \\
a_{i_{k} j_{t}}
\end{array}\right)\right| \\
& -\left(\sum_{\substack{s=1 \\
s \neq t}}^{l}\left[\left|a_{j_{t} j_{s}}\right|+\left|\left(a_{j_{t} i_{1}}, \ldots, a_{j_{t} i_{k}}\right)[A(\alpha)]^{-1}\left(\begin{array}{c}
a_{i_{1} j_{s}} \\
\vdots \\
a_{i_{k} j_{s}}
\end{array}\right)\right|\right]\right)^{\gamma} \\
& \times\left(\sum_{\substack{s=1 \\
s \neq t}}^{l}\left[\left|a_{j_{s} j_{t}}\right|+\left|\left(a_{j_{s} i_{1}}, \ldots, a_{j_{s} i_{k}}\right)[A(\alpha)]^{-1}\left(\begin{array}{c}
a_{i_{1} j_{t}} \\
\vdots \\
a_{i_{k} j_{t}}
\end{array}\right)\right|\right]\right)^{1-\gamma} .
\end{aligned}
$$

From the proof of Theorem 3, we know

$$
\begin{aligned}
& \left|\left(a_{j_{t} i_{1}}, \ldots, a_{j_{t} i_{k}}\right)[A(\alpha)]^{-1}\left(\begin{array}{c}
a_{i_{1} j_{t}} \\
\vdots \\
a_{i_{k} j_{t}}
\end{array}\right)\right| \\
& +\left(\sum_{\substack{s=1 \\
s \neq t}}^{l}\left[\left|a_{j_{t} j_{s}}\right|+\left|\left(a_{j_{t} i_{1}}, \ldots, a_{j_{t} i_{k}}\right)[A(\alpha)]^{-1}\left(\begin{array}{c}
a_{i_{1} j_{s}} \\
\vdots \\
a_{i_{k} j_{s}}
\end{array}\right)\right|\right]\right)^{\gamma} \\
& \times\left(\sum_{\substack{s=1 \\
s \neq t}}^{l}\left[\left|a_{j_{s} j_{t}}\right|+\left|\left(a_{j_{s} i_{1}}, \ldots, a_{j_{s} i_{k}}\right)[A(\alpha)]^{-1}\left(\begin{array}{c}
a_{i_{1} j_{t}} \\
\vdots \\
a_{i_{k} j_{t}}
\end{array}\right)\right|\right]\right)^{1-\gamma} \\
& <\left(P_{j_{t}}(A)-w_{t}\right)^{\gamma}\left(S_{j_{t}}(A)-w_{t}^{T}\right)^{1-\gamma} \text {. }
\end{aligned}
$$


Therefore, from (3.6) we obtain

$$
\begin{aligned}
0 & \geq\left|\lambda-a_{t t}^{\prime}\right|-P_{t}^{\gamma}(A / \alpha) S_{t}^{1-\gamma}(A / \alpha) \\
& >\left|\lambda-a_{j_{t} j_{t}}\right|-\left(P_{j_{t}}(A)-w_{t}\right)^{\gamma}\left(S_{j_{t}}(A)-w_{t}^{T}\right)^{1-\gamma} .
\end{aligned}
$$

Thus (3.4) holds.

Remark 4 By Remark 2, it is obvious that Theorem 7 improves Theorem 4 of [1].

Corollary 4 Let $A \in \mathbb{C}^{n \times n}, \alpha=\left\{i_{1}, i_{2}, \ldots, i_{k}\right\} \subseteq N_{r}(A) \cap N_{c}(A) \neq \emptyset, \alpha^{\prime}=N-\alpha=\left\{j_{1}, j_{2}, \ldots, j_{l}\right\}$, $|\alpha|<n$. Then for each eigenvalue $\lambda$ of $A / \alpha$, there exists $1 \leq t \leq l$ such that

$$
\left|\lambda-a_{j_{t j} j_{t}}\right|<P_{j_{t}}^{\gamma}(A) S_{j_{t}}^{1-\gamma} \leq \gamma P_{j_{t}}(A)+(1-\gamma) S_{j_{t}}(A)
$$

\section{A numerical example}

In this section, we show how to estimate the bounds for eigenvalues of the Schur complement with the elements of the original matrix to show the advantages of our results.

\section{Example Let}

$$
A=\left(\begin{array}{ccccc}
330 & 323 & 2 & 3 & 1 \\
321 & 328 & 3 & 1 & 2 \\
3 & 2 & 6 & 7 & 2 \\
2 & 1 & 1 & 2 & 6 \\
2 & 1 & 2 & 3 & 4
\end{array}\right), \quad \alpha=\{1,2\}
$$

If we estimate the bounds for eigenvalues of $A / \alpha$ by the elements of $A / \alpha$, there would be great computations to do. However, as

$$
\begin{aligned}
& P_{1}(A)=329 ; \quad P_{2}(A)=327 ; \quad P_{3}(A)=14 ; \quad P_{4}(A)=10 ; \quad P_{5}(A)=8 ; \\
& S_{1}(A)=328 ; \quad S_{2}(A)=327 ; \quad S_{3}(A)=8 ; \quad S_{4}(A)=14 ; \quad S_{5}(A)=11 ; \\
& w_{3}=\min \left\{\frac{\left|a_{11}\right|-P_{1}(A)}{\left|a_{11}\right|-\left|a_{12}\right|}, \frac{\left|a_{22}\right|-P_{2}(A)}{\left|a_{22}\right|-\left|a_{21}\right|}\right\} \sum_{i=1}^{2}\left|a_{3 i}\right|=\frac{5}{7} ; \\
& w_{4}=\min \left\{\frac{\left|a_{11}\right|-P_{1}(A)}{\left|a_{11}\right|-\left|a_{12}\right|}, \frac{\left|a_{22}\right|-P_{2}(A)}{\left|a_{22}\right|-\left|a_{21}\right|}\right\} \sum_{i=1}^{2}\left|a_{4 i}\right|=\frac{3}{7} ; \\
& w_{5}=\min \left\{\frac{\left|a_{11}\right|-P_{1}(A)}{\left|a_{11}\right|-\left|a_{12}\right|}, \frac{\left|a_{22}\right|-P_{2}(A)}{\left|a_{22}\right|-\left|a_{21}\right|}\right\} \sum_{i=1}^{2}\left|a_{5 i}\right|=\frac{3}{7} ; \\
& w_{3}^{T}=\min \left\{\frac{\left|a_{11}\right|-S_{1}(A)}{\left|a_{11}\right|-\left|a_{21}\right|}, \frac{\left|a_{22}\right|-S_{2}(A)}{\left|a_{22}\right|-\left|a_{12}\right|}\right\} \sum_{i=1}^{2}\left|a_{i 3}\right|=1 ; \\
& w_{4}^{T}=\min \left\{\frac{\left|a_{11}\right|-S_{1}(A)}{\left|a_{11}\right|-\left|a_{21}\right|}, \frac{\left|a_{22}\right|-S_{2}(A)}{\left|a_{22}\right|-\left|a_{12}\right|}\right\} \sum_{i=1}^{2}\left|a_{i 4}\right|=\frac{4}{5} ;
\end{aligned}
$$


Since $\alpha \in N_{r}(A)$, according to Theorem 5 , the eigenvalue $z$ of $A / \alpha$ satisfies

$$
z \in\{z|| z-6 \mid \leq 13.29\} \cup\{z|| z-2 \mid \leq 9.58\} \cup\{z|| z-4 \mid \leq 7.58\} \equiv G_{1} .
$$

According to Theorem 3 in [1], the eigenvalue $z$ of $A / \alpha$ satisfies

$$
z \in\{z|| z-6 \mid \leq 13.98\} \cup\{z|| z-2 \mid \leq 9.99\} \cup\{z|| z-4 \mid \leq 7.99\} \equiv G_{1}^{\prime} .
$$

Further, we use Figure 1 to illustrate (4.1) and (4.2).

It is clear that $G_{1} \subset G_{1}^{\prime}$ from both (4.1), (4.2) and Figure 1.

In addition, since $\alpha \in N_{r}(A) \cap N_{c}(A)$, by taking $\gamma=\frac{1}{2}$ in Theorem 7, the eigenvalue $z$ of $A / \alpha$ satisfies

$$
z \in\{z|| z-6 \mid \leq 9.64\} \cup\{z|| z-2 \mid \leq 11.24\} \cup\{z|| z-4 \mid \leq 8.87\} \equiv G_{2} .
$$

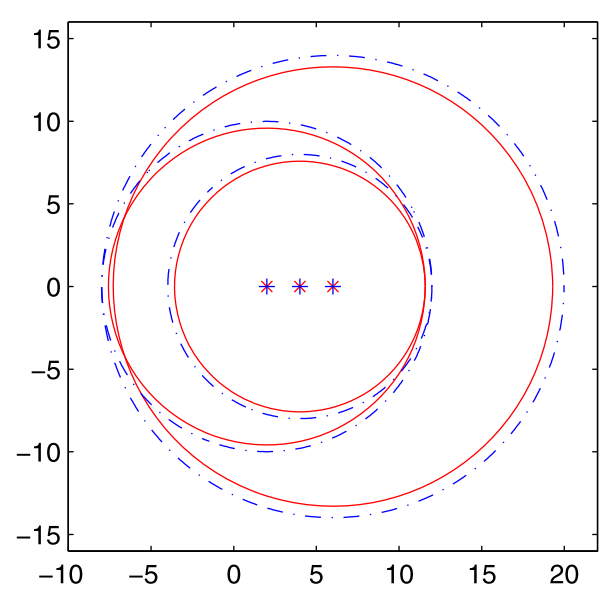

Figure 1 The red dotted line and blue dashed line denote the corresponding discs of $G_{1}$ and $G_{1}^{\prime}$, respectively.

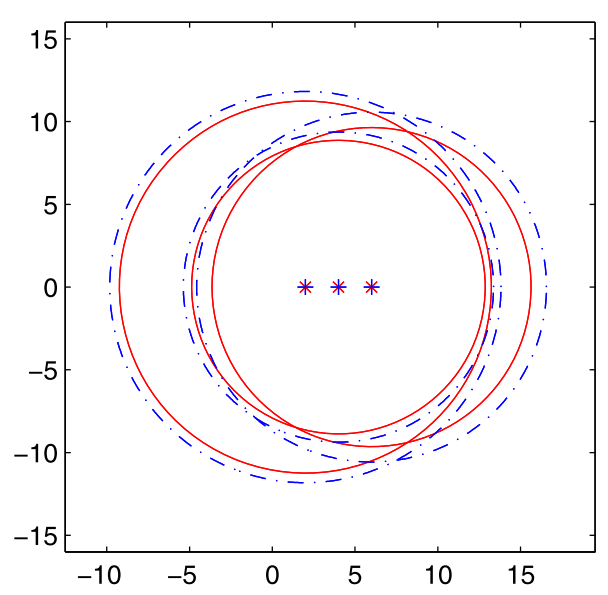

Figure 2 The red dotted line and blue dashed line denote the corresponding discs of $G_{2}$ and $G_{2}^{\prime}$, respectively. 
According to Theorem 4 in [1], the eigenvalue $z$ of $A / \alpha$ satisfies

$$
z \in\{z|| z-6 \mid \leq 10.57\} \cup\{z|| z-2 \mid \leq 11.82\} \cup\{z|| z-4 \mid \leq 9.37\} \equiv G_{2}^{\prime}
$$

Further, we use Figure 2 to illustrate (4.3) and (4.4).

It is clear that $G_{2} \subset G_{2}^{\prime}$ from both (4.3), (4.4) and Figure 2 .

\section{Competing interests}

The authors declare that they have no competing interests.

\section{Authors' contributions}

JZ and JZL carried out the preparation, participated in the sequence alignment and drafted the manuscript. GT participated in its design and coordination. All authors read and approved the final manuscript.

\section{Acknowledgements}

The work was supported in part by the National Natural Science Foundation of China (10971176), the Program for Changjiang Scholars and Innovative Research Team in University of China (No. IRT1179), the Key Project of Hunan Provincial Natural Science Foundation of China (10JJ2002), the Key Project of Hunan Provincial Education Department of China (12A137), the Hunan Provincial Innovation Foundation for Postgraduate (CX2011B242) and the Aid Program Science and Technology Innovative Research Team in Higher Educational Institutions of Hunan Province of China.

Received: 14 October 2011 Accepted: 14 December 2012 Published: 3 January 2013

\section{References}

1. Liu, JZ, Huang, ZJ: The Schur complements of $\gamma$-diagonally and product $\gamma$-diagonally dominant matrix and their disc separation. Linear Algebra Appl. 432, 1090-1104 (2010)

2. Liu, JZ, Huang, ZJ, Zhang, J: The dominant degree and disc theorem for the Schur complement. Appl. Math. Comput. 215, 4055-4066 (2010)

3. Xiang, SH, Zhang, SL: A convergence analysis of block accelerated over-relaxation iterative methods for weak block $H$-matrices to partition $\pi$. Linear Algebra Appl. 418, 20-32 (2006)

4. Horn, RA, Johnson, CR: Topics in Matrix Analysis. Cambridge University Press, New York (1991)

5. Smith, R: Some interlacing properties of the Schur complement of a Hermitian matrix. Linear Algebra Appl. 177 137-144 (1992)

6. Carlson, D, Markham, T: Schur complements on diagonally dominant matrices. Czechoslov. Math. J. 29(104), 246-251 (1979)

7. Ikramov, KD: Invariance of the Brauer diagonal dominance in Gaussian elimination. Moscow. Univ. Comput. Math Cybern. 2, 91-94 (1989)

8. Li, B, Tsatsomeros, M: Doubly diagonally dominant matrices. Linear Algebra Appl. 261, 221-235 (1997)

9. Golub, GH, Van Loan, CF: Matrix Computations, 3rd edn. Johns Hopkins University Press, Baltimore (1996)

10. Kress, R: Numerical Analysis. Springer, New York (1998)

11. Liu, JZ, Li, JC, Huang, ZH, Kong, X: Some properties on Schur complement and diagonal-Schur complement of some diagonally dominant matrices. Linear Algebra Appl. 428, 1009-1030 (2008)

12. Liu, JZ, Huang, YQ: The Schur complements of generalized doubly diagonally dominant matrices. Linear Algebra Appl. 378, 231-244 (2004)

13. Liu, JZ, Huang, YQ: Some properties on Schur complements of $H$-matrices and diagonally dominant matrices. Linear Algebra Appl. 389, 365-380 (2004)

14. Liu, JZ, Zhang, FZ: Disc separation of the Schur complements of diagonally dominant matrices and determinantal bounds. SIAM J. Matrix Anal. Appl. 27, 665-674 (2005)

15. Liu, JZ: Some inequalities for singular values and eigenvalues of generalized Schur complements of products of matrices. Linear Algebra Appl. 286, 209-221 (1999)

16. Liu, JZ, Zhu, L: A minimum principle and estimates of the eigenvalues for Schur complements of positive semidefinite Hermitian matrices. Linear Algebra Appl. 265, 123-145 (1997)

17. Liu, JZ, Zhang, J, Liu, Y: The Schur complement of strictly doubly diagonally dominant matrices and its application. Linear Algebra Appl. 437, 168-183 (2012)

doi:10.1186/1029-242X-2013-2

Cite this article as: Zhang et al.: The improved disc theorems for the Schur complements of diagonally dominant matrices. Journal of Inequalities and Applications 2013 2013:2. 